\title{
The LHCb VELO for Phase 1 Upgrade
}

\section{Cameron Dean*, on behalf of the LHCb Collaboration}

University of Glasgow

E-mail: cameron.deandecern.ch

Large Hadron Collider beauty (LHCb) is a dedicated experiment for studying $b$ and $c$ hadrons at the Large Hadron Collider (LHC). LHCb uses a silicon-strip detector, the Vertex Locator (VELO), for high precision tracking of collisions from the LHC. During Long Shutdown 2 (LS2) of the $\mathrm{LHC}$, the LHCb collaboration will upgrade the detector, switching from the current VELO, capable of a $1 \mathrm{MHz}$ readout, to a hybrid pixel detector capable of reading out at the full bunch crossing rate of the LHC. Substantial progress has been made in the development of the new detector. The status of the silicon sensors, custom designed VeloPix ASIC and electronic system will be discussed in detail. The current status of the cooling system and RF foil will also be presented.

The 25th International Workshop on Vertex Detectors

September 26-30, 2016

La Biodola, Isola d'Elba, ITALY

${ }^{*}$ Speaker. 


\section{Introduction}

LHCb is a single arm spectrometer [1] optimised to study the decays of $b$ and $c$ hadrons covering a pseudorapidity $(\eta)$ region of $2<\eta<5$ To help with the physics goal, the experiment runs at a lower luminosity than the other large experiments at the LHC, with an output rate from the hardware trigger of $\sim 1 \mathrm{MHz}$. The VELO surrounds the interaction region and consists of an array of silicon-strip sensors with a pitch of $40-100 \mu \mathrm{m}$ with the first sensitive strip at $8.2 \mathrm{~mm}$ from the beam [2]. As this distance is deemed to be unsafe outside stable beams, the VELO retracts to $29 \mathrm{~mm}$ when not taking physics data. The VELO is contained within a secondary vacuum, separated from the main beam by a $300 \mu \mathrm{m}$ RF foil and is cooled by $\mathrm{CO}_{2}$, keeping the silicon strips at an operational temperature of $\sim-10{ }^{\circ} \mathrm{C}$.

During LS2, LHCb will undergo a full upgrade. The hardware trigger will be removed and the detector will be read out at the full bunch crossing frequency of up to $40 \mathrm{MHz}$ which requires the alteration of all the front end electronics. After the upgrade, the peak luminosity in LHCb will increase by a factor of five over the current luminosity to $4 \times 10^{33} \mathrm{~cm}^{-2} \mathrm{~s}^{-1}$ which will increase the number of visible interactions $(\mu)$ from 1.2 to 5.2 as can be seen in Figure 1 [3]. To cope with the new conditions the current VELO will be replaced with a hybrid pixel detector with a pixel pitch of $55 \mu \mathrm{m}$, the distance from the beam to the closest pixel will be reduced to $5.1 \mathrm{~mm}$ and the RF foil will be changed to a thickness of $250 \mu \mathrm{m}$. The upgraded VELO will operate in a harsher radiation environment thus the radiation hardness will be improved to withstand a dose of $8 \times 10^{15} 1 \mathrm{MeV} n_{e q} \mathrm{~cm}^{-2}$ compared to the current requirement of $7 \times 10^{14} 1 \mathrm{MeV} \mathrm{n}_{e q} \mathrm{~cm}^{-2}$ [4]. An upgraded VELO module will consist of a $200 \mu \mathrm{m}$ silicon sensor bump bonded to the custom designed VeloPix ASIC [5] and will be actively chilled by evaporative $\mathrm{CO}_{2}$ microchannel cooling. The design of the upgrade can be seen in Figure 2.
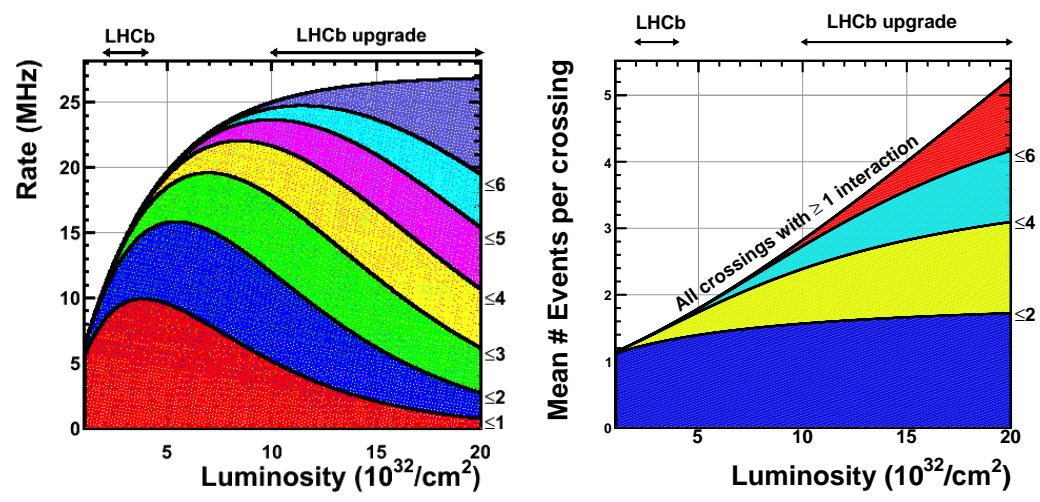

Figure 1: (left) Evolution of the interaction rate expected in LHCb as a function of the luminosity, split into bands of number of interactions per bunch crossing. (right) Evolution of the pileup as a function of the luminosity.

\section{Physics Motivation}

The new VELO design gives several physics advantages over the current design. The reduced material budget and distance from the beam significantly improves the Impact Parameter resolution 

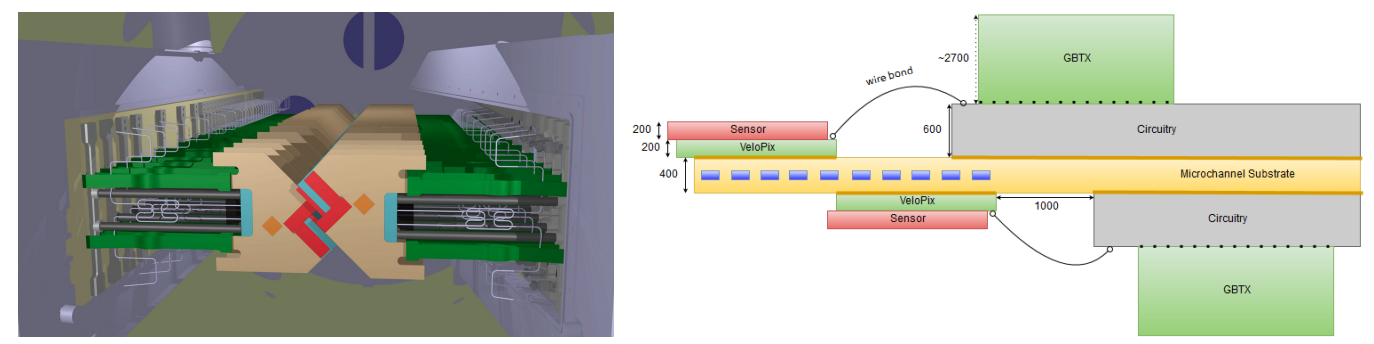

Figure 2: (left) Rendering of the VELO upgrade in the closed position. (right) Side view of a VELO Upgrade module [4].

$\left(\sigma_{I P}\right)$ at low values of transverse momentum $\left(p_{T}\right)$ as can be seen in Figure 3. The $\sigma_{I P}$ at high $p_{T}$ is comparable for both the current VELO and the upgrade due to the increased pitch of the pixels. There is also an improved efficiency with $\varepsilon>99 \%$ across most regions, which is seen in Figure 3 , and a reduced ghost rate; from $6.2 \%$ in the current VELO at $\mu=1.2$ to $2.5 \%$ at $\mu=5.2$ for the upgrade. A significant change is seen in the $\phi$ efficiency due to the change from silicon strips to pixels and the L-shaped geometry avoiding the reduced efficiency present in the overlap regions of the current VELO.

\section{Sensor Studies}

The upgrade sensors will consist of $200 \mu \mathrm{m}$ thick silicon bump-bonded to three custom designed ASICs, the VeloPix, and will have dimensions of $14 \times 43 \mathrm{~mm}$. The nominal implant width is $39 \mu \mathrm{m}$ with a guard ring width of $450 \mu \mathrm{m}$. Prototype sensors have been produced by Micron (who provided sensors with a $36 \mu \mathrm{m}$ implant) and Hamamatsu and extensively tested before and after irradiation to half $\left(4 \times 10^{15} 1 \mathrm{MeV} n_{e q} \mathrm{~cm}^{-2}\right)$ and the full expected fluence $\left(8 \times 10^{15} 1 \mathrm{MeV} n_{e q} \mathrm{~cm}^{-2}\right)$.

Sensor testing was conducted using the Timepix3 Telescope at CERN's Super Proton Synchrotron (SPS). The telescope consists of 8 Timepix3 ASICs [6] bump bonded to silicon sensors with a device under test (DUT) in the centre capable of $\mathrm{x}, \mathrm{y} \& \theta$ motion. The telescope has a resolution of $2 \mu \mathrm{m}$ with a hit rate of 80 Mhits/s using a $180 \mathrm{GeV}$ beam.

The sensors are required to withstand $1000 \mathrm{~V}$ bias without signs of breakdown after receiving the full fluence and produce a signal of $6000 \mathrm{e}^{-}$to maintain a good $\mathrm{S} / \mathrm{N}$ ratio. Several sensors were tested and have shown that they achieve the required $6000 \mathrm{e}^{-}$below the $1000 \mathrm{~V}$ limit imposed with no breakdown after the full irradiation. Sensors that were exposed to half the fluence were tested and shown to collect $6000 \mathrm{e}^{-}$at a lower bias than the fully irradiated sensors as expected. The results from measurements of prototype sensors are seen in Figure 4

As the telescope has a resolution of $\sim 2 \mu \mathrm{m}$ it is possible to obtain the intrapixel efficiency by extrapolating the tracks position from the arms of the telescope while looking for a hit in the corresponding pixel of the DUT then superimposing all the pixels on top of each other. With a pixel size of $55 \times 55 \mu \mathrm{m}$ this allows the efficiency to be split into a $25 \times 25$ matrix. After full irradiation, at low voltages the pixels showed a large drop in efficiency at the edges due the distance of the track from the implant requiring the charge to travel further. High efficiency was recovered when the bias was increased towards $1000 \mathrm{~V}$. The results from some of the sensors are shown in Figure 5 . 

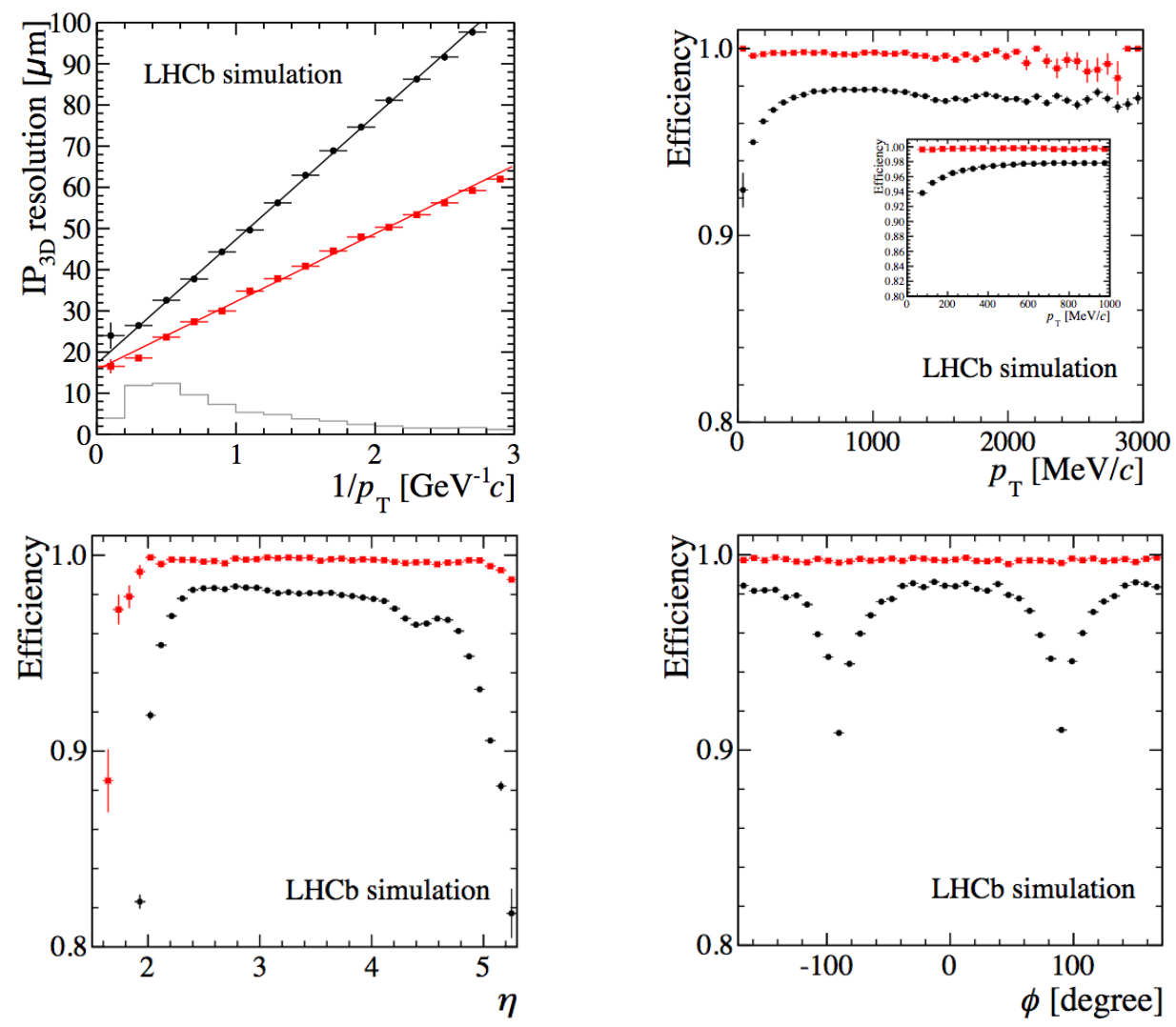

Figure 3: (top left) Comparison of $\sigma_{I P}$ for the current VELO (in black) and the upgrade (in red). The histogram shows the relative population of events in each bin. (top right) Efficiency vs $p_{T}$ (the smaller plot shows a higher resolution of the low $p_{T}$ region). (bottom left) Efficiency vs $\eta$. (bottom right) Efficiency vs Track Angle. The plots are produced from detector simulations in upgrade conditions with $\mu=5.2$ and $\sqrt{s}=14 \mathrm{TeV}$.

The DUT is capable of rotating with respect to the beam hence it is possible to obtain the sensor resolution as a function of beam angle. This is obtained from the standard deviation of the residual between the cluster in the DUT and the position of the track from the arms of the telescope. Figure 6 shows that the resolution of the sensors degrades after irradiation, caused by charge trapping.

\section{The VeloPix}

The VELO upgrade will use a custom designed ASIC, the VeloPix, to handle the huge data rate that will be produced (up to $15.1 \mathrm{Gbit} / \mathrm{s}$ from the hottest ASIC) and is based on the Timepix3 that has been used to characterise the sensors in testbeam. The VeloPix has four serial data outputs which send data at a constant rate of $5.12 \mathrm{Gbit} / \mathrm{s}$. Each hit is time-stamped with the LHC bunch crossing number. The VeloPix has been delivered and initial tests have shown that the ASIC works to the required specifications [5]. A comparison of the key features and difference between the two chips is presented in Table 1 

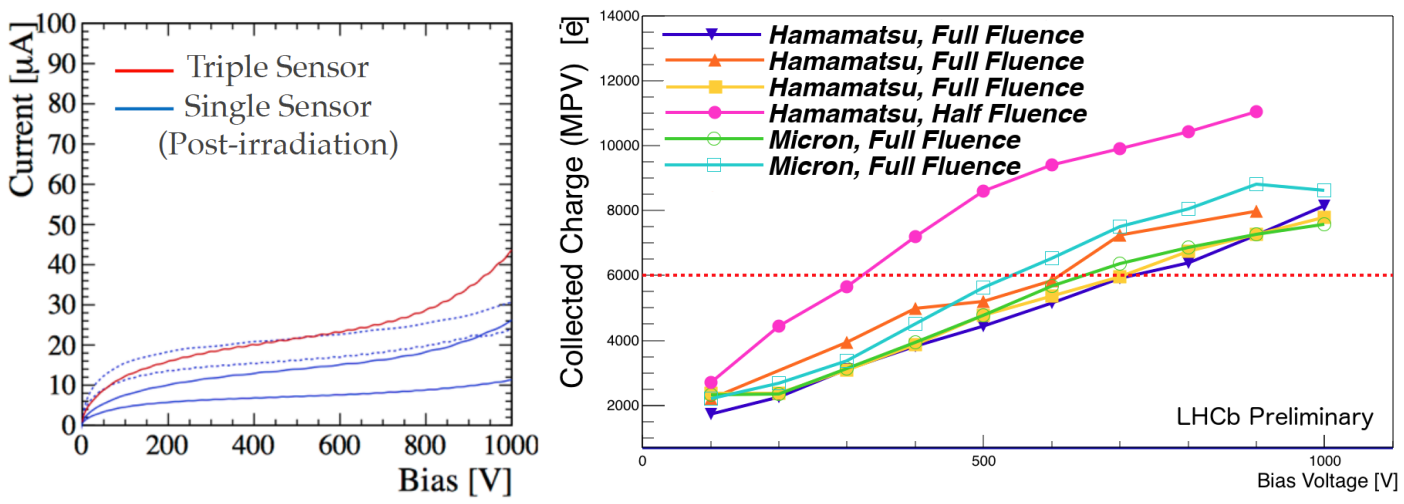

Figure 4: (left) IV scan from fully irradiated sensors (a Single Sensor is one which covers a single ASIC while a Triple Sensor covers three ASICs). (right) Charge collection results from a variety of irradiated sensors. The $6000 \mathrm{e}^{-}$requirement is marked with the dashed line. The pink plot with filled circles is from a sensor that had been exposed to half the expected fluence, the others are irradiated to the full expected fluence.
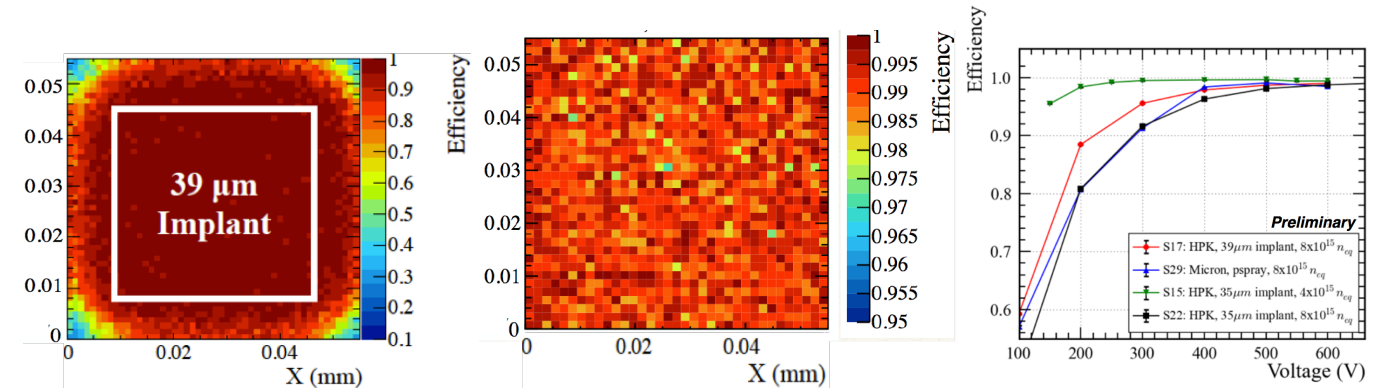

Figure 5: (left) The intrapixel efficiency for a Hamamatsu sensor at $300 \mathrm{~V}$ after being exposed to the full expected fluence. (middle) The intrapixel efficiency for the same sensor at $1000 \mathrm{~V}$. (right) The average efficiency for 3 fully irradiated sensors and 1 sensor with half the irradiation (green with triangles)

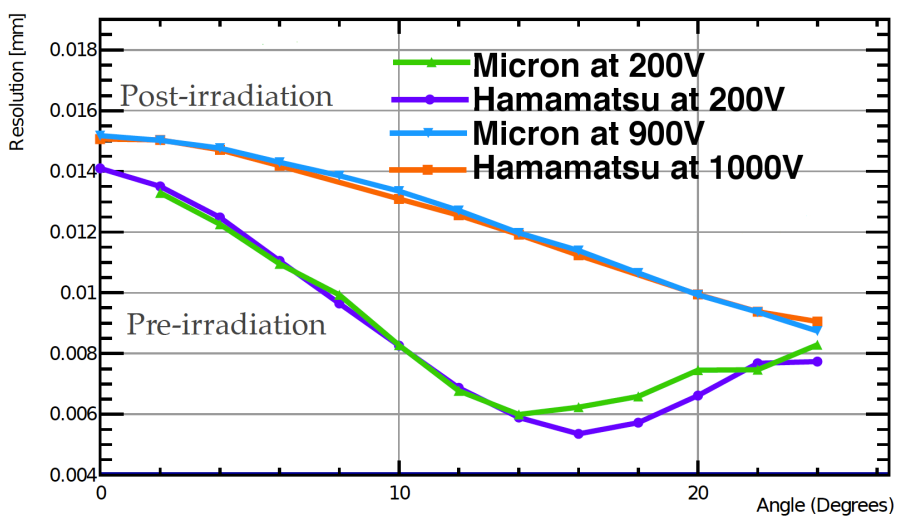

Figure 6: The resolution of sensors before and after irradiation as a function of beam angle. Purple Pre-irradiation Hamamatsu, Green - Pre-irradiation Micron, Orange - Post-irradaition Hamamatsu, Blue Post-irradiation Micron. 
Table 1: Comparison of the key features and differences between Timepix3 and VeloPix.

\begin{tabular}{ccc}
\hline Feature & Timepix3 & VeloPix \\
\hline Readout & ToT & Binary \\
Max. Power & $1 \mathrm{~W} / \mathrm{cm}^{2}$ & $1.5 \mathrm{~W} / \mathrm{cm}^{2}$ \\
Pixel Matrix $/$ Size & $256 \times 256 / 55 \mu \mathrm{m} \times 55 \mu \mathrm{m}$ & $256 \times 256 / 55 \mu \mathrm{m} \times 55 \mu \mathrm{m}$ \\
Hit Rate & $80 \mathrm{Mhit} / \mathrm{s}$ & $900 \mathrm{Mhit} / \mathrm{s}$ \\
Data Rate & $5.12 \mathrm{Gbit} / \mathrm{s}$ & $20.48 \mathrm{Gbit} / \mathrm{s}(4 \times 5.12)$ \\
Technology & $130 \mathrm{~nm} \mathrm{CMOS}$ & $130 \mathrm{~nm} \mathrm{CMOS}$ \\
\hline
\end{tabular}

\section{Electronics}

The data output of the VELO presents a significant challenge to the upgrade with individual ASICs predicted to produce a peak data rate of $15.1 \mathrm{Gbit} / \mathrm{s}$ and the full detector producing up to $2.85 \mathrm{Tbit} / \mathrm{s}$ [4]. The data will be carried from the VELO via optical links but due to the issues with having electrical to optical conversion components within the harsh radiation environment near the collision point, the increased power consumption and the components becoming inaccessible during operation, the conversion will take place outside the vacuum of the VELO. This requires the development of radiation hard electrical components that can also handle the motion of the VELO without any loss in signal.

The upgrade electronic system consists of four distinct parts; front-end hybrids mounted on the modules that distribute power and control signals to and route the serial data from the VeloPix ASICs, high speed flex-links to carry the signal from the modules to the vacuum wall and absorb the motion of the VELO, vacuum feedthroughs to pass the signal through the vacuum wall and opto-power boards (OPB) for optical/electrical conversion and to provide local voltage control. These components will be exposed to different levels of irradiation over the lifetime of the detector with the data links receiving a dose of up to $2 \times 10^{13} 1 \mathrm{MeV} n_{e q} \mathrm{~cm}^{-2}$ and the OPB receiving a dose an order of magnitude lower. The serial links are implemented as edge-coupled micro strips and are produced using low loss high-speed qualified dielectrics ${ }^{1}$.

The electronics were designed to give a standard impedance of $100 \Omega$ with individual components having a signal loss of around $3 \mathrm{~dB}$ and a full system loss of less than $10 \mathrm{~dB}$. The trace widths were optimised to reduce the signal attenuation while still retaining the $100 \Omega$ differential impedance, where the flexible data tapes have a trace width and gap of $200 \mu \mathrm{m}$ and the rigid PCBs have a trace width and gap of $150 \mu \mathrm{m}$. The flexible data tape has seven parallel links which are required to transfer data from the hottest tile. The electronic prototypes were tested at the Nyquist frequency of the data rate of a single VeloPix channel $(2.5 \mathrm{GHz})$ [7] to investigate their bit-error rates (BER) and eye diagrams using a real time oscilloscope and a serial data source. The prototypes were also tested up to $13.5 \mathrm{GHz}$ to investigate the signal loss and impedance using a vector network analyser (VNA).

The signal attenuation at the Nyquist frequency for the different parts of the system are summarised in Table 2 and the differential transmission for the link and its components are shown in

\footnotetext{
${ }^{1}$ The flex circuits were produced using Pyralux AP8575 and the rigid circuits were produced using I-Tera
} 
Table 2: Signal losses (in dB) across different components of the electrical system at $2.5 \mathrm{GHz}$. The channels on the OPB and vacuum feedthrough consist of parallel traces and show good consistency with one another.

\begin{tabular}{cc}
\hline Component & Loss at 2.5 GHz [dB] \\
\hline Full System & 9.37 \\
Hybrid & 2.28 \\
Flexible Data Tape & 3.94 \\
OPB \& Vacuum Feedthrough, Channel 1 & 3.07 \\
OPB \& Vacuum Feedthrough, Channel 2 & 4.55 \\
OPB \& Vacuum Feedthrough, Channel 3 & 3.35 \\
OPB \& Vacuum Feedthrough, Channel 4 & 4.15 \\
OPB \& Vacuum Feedthrough, Channel 5 & 3.21 \\
OPB \& Vacuum Feedthrough, Channel 6 & 3.02 \\
\hline
\end{tabular}

Figure 7, each individual component was measured to have losses close to the $3 \mathrm{~dB}$ design specification with the full system giving a loss of around $9.4 \mathrm{~dB}$. The differential impedance of the system was also determined from analysis of the data from the VNA, emulating a time-domain reflectometry measurement. It was found to be near the $100 \Omega$ design specification for all the components (Figure 8) with linear increases in the impedance produced from DC resistance. The eye diagrams were measured using a $5.12 \mathrm{Gbit} / \mathrm{s}$ pseudo-random 7-bit pattern (PRBS7) with individual components showing fully open eyes.

As the VELO moves, the flex-links are required to absorb the motion. Hence it is important to know that the links are capable of repeated bending without a significant loss in signal. A device was created to simulate the motion the tape would experience in operation and was bent 3000 times (calculated as the likely number of bends a link would receive over its lifetime) with no apparent loss in signal as shown in Figure 9.

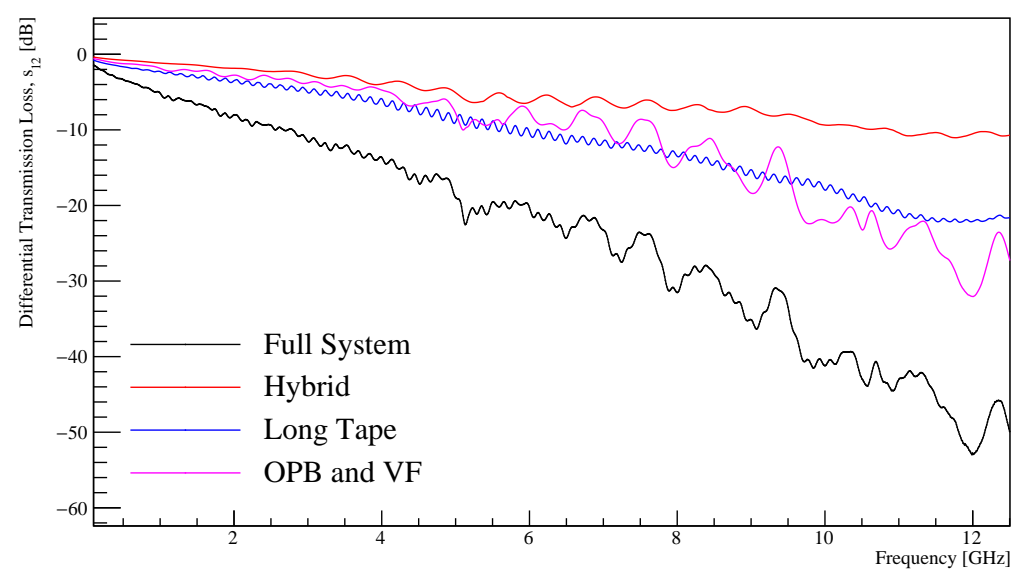

Figure 7: Losses for the full electrical system (black), front-end hybrid (red), flexible data tape (blue) and combined vacuum feedthrough and OPB (magenta). 


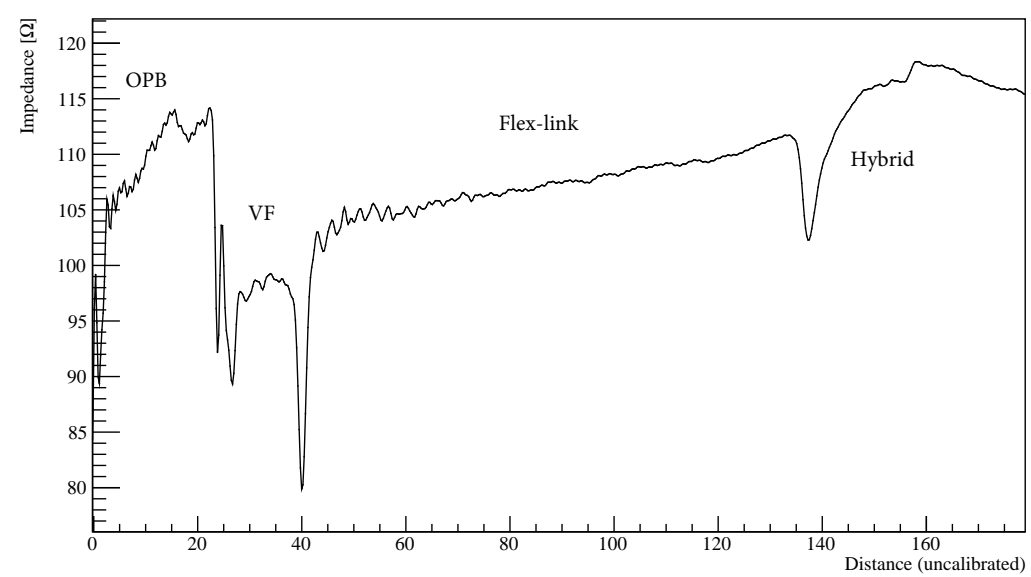

Figure 8: Differential impedance of the electrical components in the full system.

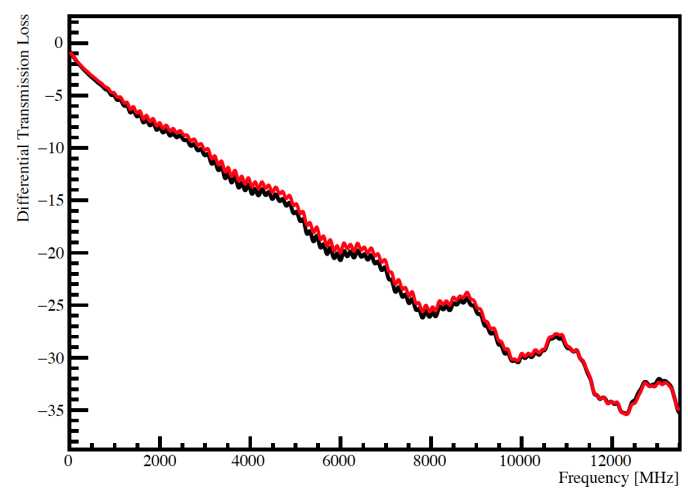

Figure 9: Measured signal losses in a flex-link before (black) and after (red) 3000 bends.

\section{Cooling and RF Foil}

To avoid thermal runaway and radiation damage in the VELO, the sensors will be kept below $-20{ }^{\circ} \mathrm{C}$. To achieve this the modules will use evaporative $\mathrm{CO}_{2}$ microchannel cooling with the channels being etched into a silicon substrate to avoid mismatch between the coefficient of thermal expansion (CTE) and allow direct cooling.The location of the microchannels within the module can be seen in Figure 2. The etching will produce $120 \mu \mathrm{m}$ deep trenches with $140 \mu \mathrm{m}$ of silicon on either side of the trench to evenly cool both sides of the module and optimise the strength of the substrate.

The VeloPix are expected to produce up to $1.5 \mathrm{~W} / \mathrm{cm}^{2}$ so the cooling is expected to be able to dissipate up to $12 \mathrm{~W}$ under normal conditions with an input $\mathrm{CO}_{2}$ temperature of $-30{ }^{\circ} \mathrm{C}$ and be able to have a pressure tolerance of 186 bar.

The RF foil that separates the VELO from the LHC beam constitutes the majority of the material encountered by a particle before the second measured point (Figure 10), hence it is necessary to make it as thin as possible. Small scale prototype models milled from a single block of aluminium have been produced down to a thickness of $250 \mu \mathrm{m}$ and will sit $3.5 \mathrm{~mm}$ from the beam. 


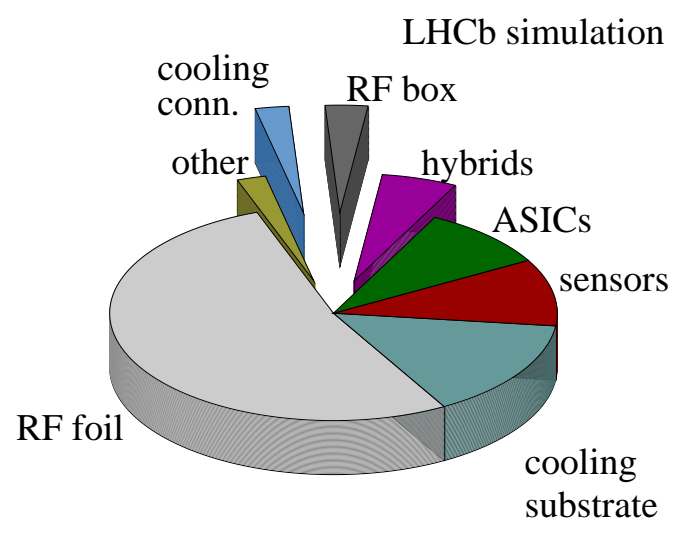

total material: $21.3 \% X_{0}$

Figure 10: Distribution of the material budget in the VELO Upgrade.

\section{Conclusion}

After LS2, the luminosity in LHCb will increase by a factor five over the current operating conditions. To cope with this new challenge most of the current detector will be upgraded and the hardware trigger will be removed. The VELO upgrade constitutes one of the most significant changes, going from a silicon strip detector with a $1 \mathrm{MHz}$ readout to a hybrid pixel detector capable of reading out the full $40 \mathrm{MHz}$ bunch crossing rate of the LHC. The project is in a good state of development with prototypes of all subcomponents having been delivered and tested. Extensive testing of the sensor and electronic prototypes have been conducted with further components performing to the desired specifications keeping the project on track to installation after the completion of Run II of the LHC.

\section{References}

[1] The LHCb Collaboration, The LHCb Detector at the LHC, Journal of Instrumentation 2008 3-08 S08005

[2] The LHCb VELO Group, Performance of the LHCb Vertex Locator, Journal of Instrumentation 2014 9 P09007

[3] The LHCb Collaboration, Framework TDR for the LHCb Upgrade, CERN, 2012

[4] The LHCb Collaboration, LHCb VELO Upgrade Technical Design Report, CERN, 2013

[5] T. Poikela, The VeloPix ASIC, Journal of Instrumentation, Proceeding from TWEPP 2016

[6] M. van Beuzekom et al., Timepix3: a 65K Channel Hybrid Pixel Readout Chip with Simultaneous ToA/ToT and Sparse Readout, Journal of Instrumentation 2014 9-05 C05013

[7] F. L. Luo et al., Digital Power Electronics and Applications, Academic Press, 2005 